\title{
Evaluación de la madurez de la gestión de la seguridad y salud en el trabajo: revisión de literatura*
}

\section{Maturity of safety and health at work management assessment: Review of literature}

\section{Avaliação da maturidade da segurança e saúde no trabalho: Revisão de literatura}

Recibido: 15 de mayo de 2017 Revisado: 23 de junio de 2017

Aceptado: 28 de junio de 2017

Yuber Liliana Rodríguez-Rojas**

Convenio Universidad Santo Tomás - Icontec, Universitaria Agustiniana

Ximena Lucía Pedraza-Najar ${ }^{*} * *$ *

CORPOEDUCAD, Publimark Eduinternational

Jaime Apolinar Martínez Arroyo*****

Universidad Michoacana de San Nicolás de Hidalgo - México

* Artículo de revisión. Muestra los resultados parciales de una investigación doctoral. DOI: http://dx.doi.org/10.15332/s2145-1389.2017.0001.08

* Doctora (c) en Administración. Magíster en Salud y Seguridad en el Trabajo. Fisioterapeuta. Líder de la línea de investigación en Gestión en Salud. Docente investigadora, Convenio Universidad Santo Tomás -Icontec. Docente de posgrados de la Universitaria Agustiniana. Correo electrónico: yuberliliana@gmail.com.

** Doctora (c) en Administración. Magíster en Calidad y Gestión Integral. Especialista en Gestión de la Producción, la Calidad y la Tecnología. Especialista en Gerencia de Procesos, Calidad e Innovación. Microbióloga. Investigadora de la línea de Educación de la Corporación para la Administración y el Desarrollo Socioeducativo CORPOEDUCAD y gerente general de Publimark Eduinternational. Correo electrónico: ximenapedraza@yahoo.com.

**** Doctor en Ciencias en Negocios Internacionales. Profesor investigador de la Facultad de Contaduría y Ciencias Administrativas de la Universidad Michoacana de San Nicolás de Hidalgo. Correo electrónico: corredor42195@hotmail.com. 


\section{RESUMEN}

Este artículo tiene como finalidad presentar los resultados de una revisión de literatura cuyo propósito fue identificar variables y dimensiones para la medición de la madurez de la gestión de la seguridad y salud en el trabajo. La revisión de la literatura realizada sobre 38 artículos publicados entre 2001-2017 en ScienceDirect, EBSCO, Eric y Redalyc y 6 documentos detectados a través de búsqueda iterativa, permitió identificar 10 dimensiones agrupadas en 3 variables que pueden contribuir en la medición de la madurez de esta gestión, a saber: contexto interno, contexto externo y el enfoque de la seguridad y salud en el trabajo (concebido como estrategia central).

Palabras clave: seguridad y salud en el trabajo, gestión de la seguridad y salud ocupacional, aprendizaje organizacional, evaluación de la madurez, criterios de medición.

\section{ABSTRACT}

This article aims to present the results of a review of the literature whose purpose identified variables and dimensions for the measurement of the maturity of safety and health at work management. Safety and health at work management has been modified in response to the current dynamics of organizations due to the introduction of new schemes of work, which are products of the development of the global economy. The review of the literature on 38 articles published between 2001-2017 in ScienceDirect, EBSCO, Eric and Redalyc and 6 documents detected through iterative search, which allowed to identify 10 dimensions grouped into 3 variables that can contribute to the measurement of the maturity of this management. These are internal context, external context and occupational safety and health approach (conceived as central strategy).

Keywords: Safety and health at work, safety and occupational health management, organizational learning, maturity evaluation, assessment criteria.

\section{RESUMO}

Este artigo tem como objetivo apresentar os resultados de uma revisão de literatura cujo objetivo foi identificar variáveis e dimensões para medir a maturidade da Gestão de Segurança e Saúde no Trabalho. A revisão da literatura realizada em 38 artigos publicados entre 2001-2017, em Science Direct, EBSCO, Eric e Redalyc e 6 documentos detectados através de uma pesquisa iterativa, permitiu identificar 10 dimensões agrupadas em 3 variáveis que podem contribuir na medição da maturidade desta gestão, a saber: contexto interno, contexto externo e o enfoque da segurança e saúde no trabalho, (concebido como uma estratégia central).

Palavras-chave: segurança e saúde no trabalho, gestão da segurança e saúde ocupacional, aprendizagem organizacional, avaliação de maturidade, critérios de medição.

\section{INTRODUCCIÓN}

Los sistemas de Gestión de la Seguridad y Salud en el Trabajo (GSST) se han proliferado en las últimas tres décadas, aspecto que ha llevado a masificar el uso de las directrices propuestas por la Organización Internacional del Trabajo (OIT) y de la norma OHSAS 18001-Occupational Health and Safety Management; si bien estas fuentes brindan lineamientos para la 
formulación, implementación y evaluación de la GSST, es necesario desarrollar nuevos métodos de evaluación que den cuenta del nivel de madurez de la misma. Cabe anotar, que el concepto "modelo de madurez" fue desarrollado por primera vez por el Instituto de Ingeniería de Software en 1993 como un mecanismo para mejorar el desarrollo y el mantenimiento de software (Kluth, Jäger, Schatz y Bauernhansl, 2014).

El concepto de modelo de madurez en SST surgió en investigación de la gestión de la seguridad y ha sido aplicada al desarrollo de la cultura de seguridad en industrias de "alto riesgo", entre ellas, industrias petroquímicas, marítimas, ferroviarias y aéreas. Se originó en modelos de calidad organizacional y en modelos de la industria del software. Fueron creados para que las organizaciones comprendieran su propio nivel de madurez en la gestión de la seguridad, mediante la evaluación del nivel de cumplimiento de los diversos elementos clave de la cultura de la seguridad a través de una serie de etapas (generalmente 5 etapas) (Foster y Hoult, 2013; Chen, Yu y Zheng, 2014).

En términos generales, la madurez significa que algo está totalmente desarrollado o perfeccionado. Para dar cuenta de ello se crearon modelos, los cuales indican las capacidades (competencia específica que debe existir en una organización con el fin de que se ejecuten procesos de gestión de proyectos y entregar servicios de gestión de proyectos y productos) de una organización. Estas capacidades se presentan de forma agrupada y describen generalmente el grado de coherencia, la visibilidad y el control dentro de la organización. Los modelos transitan de un nivel bajo, también conocido como "ad hoc" o "reactivo" a un nivel alto, en el cual se incorporan procesos de realimentación y de mejora continua. Asimismo, para conocer la existencia de la capacidad es necesario efectuar una verificación y medición de los resultados (Tahri y Drissi-Kaitouni, 2015). En este sentido, el desarrollo de la madurez es un proceso continuo dentro de las organizaciones (Ferreira y Simões, 2015).

En el campo de la GSST el reconocimiento de la cultura organizacional y su relación con los estilos de GSST es un tema reciente (Cliff, 2012), en la actualidad se estudia la influencia de la cultura de la empresa sobre el desempeño de SST a través de modelos de madurez de la GSST. En relación con la evaluación de la GSST se ha tenido pocos avances investigativos y los desarrollos hasta la fecha se han concentrado en auditorias, indicadores de desempeño y cumplimiento legal; por ello, en este artículo se presentan los resultados de una revisión de literatura cuyo propósito fue identificar variables y dimensiones para la medición de la madurez de esta gestión.

\section{METODOLOGÍA}

La revisión de la literatura fue realizada sobre 160 artículos publicados entre 2004-2016 en ScienceDirect, EBSCO, Eric y Redalyc; sin embargo, fueron incluidos en el análisis 38 artículos, los cuales aportaban en la comprensión de la variable madurez de la gestión de la seguridad y salud en el trabajo. La búsqueda se realizó con los términos clave maturity, occupational health and safety management, occupational health and safety management systems, safety and health at work. La mayoría de los artículos detectados se han publicado entre 2011 y 2017, aspecto que evidenció que la madurez de la gestión de la seguridad y salud en el trabajo se ha abordado desde investigaciones principalmente en la última década.

Estos documentos fueron revisados utilizando la técnica de análisis de contenido para detectar las dimensiones de la madurez de la gestión de la seguridad y salud en el trabajo. Se determinó que 38 artículos cumplían con los requisitos de inclusión en el estudio. Además, 
se vincularon 6 documentos, identificados a través de búsqueda iterativa, los cuales corresponden a libros y tesis que tienen relación directa con las variables objeto de investigación. El análisis de los documentos fue efectuado por dos investigadores de manera independiente, aspecto que brindó mayor objetividad en el proceso.

\section{RESULTADOS Y DISCUSIÓN}

Tras el análisis de los artículos se encontró que son tres las variables que influyen en la madurez de la GSST. Estas variables a su vez contienen 10 dimensiones a medir, las cuales pueden dar cuenta del estado de madurez en la GSST en una organización (tabla 1).

Tabla 1. Variables y dimensiones que influyen en la madurez de la GSST

\begin{tabular}{|c|c|c|c|}
\hline Variables & Dimensiones & $\begin{array}{c}\text { Número de artículos } \\
\text { asociados a la dimensión }\end{array}$ & Estudios de soporte \\
\hline \multirow{4}{*}{ 1. Contexto interno } & a) Liderazgo de la alta dirección & 5 & $\begin{array}{l}\text { Bowen \& Ostroff, 2004; Chiang, Gómez \& } \\
\text { Salazar, 2014; Hossain, Hossain, Tarannum \& } \\
\text { Chowdhury, 2015. } \\
\text { Yorio, Willmer \& Moore, } 2015 . \\
\text { Samad, Reaburn, Davis \& Ahmed, } 2015 .\end{array}$ \\
\hline & b) Participación y comunicación & 6 & $\begin{array}{l}\text { Fang, El-Tawil \& Aguirre, } 2016 . \\
\text { Fekete, 2012. } \\
\text { Figueras, 2013. } \\
\text { Grillo, 2013. } \\
\text { Hedlund, Gummesson, Rydell \& Andersson, } \\
2016 . \\
\text { Živkovic, 2013. }\end{array}$ \\
\hline & $\begin{array}{l}\text { c) Aprendizaje y desarrollo de la } \\
\text { cultura de los individuos }\end{array}$ & 7 & $\begin{array}{l}\text { Drupsteen \& Wybo, 2015; Schnake, 2007; } \\
\text { Martins \& Coetzee, 2007; } \\
\text { Martins \& Nel, } 2014 \\
\text { Nowacki \& Bachnik, 2016; } \\
\text { Warszawska \& Kraslawski, 2015; } \\
\text { Weick \& Sutcliffe, 2015. }\end{array}$ \\
\hline & $\begin{array}{l}\text { d) Innovación, medición y } \\
\text { mejora continua }\end{array}$ & 10 & $\begin{array}{l}\text { Bernardo, 2014; } \\
\text { Carlucci, 2010; } \\
\text { Maier, Vadastreanu, Keppler, Eidenmuller \& } \\
\text { Maier, 2015; } \\
\text { Mansour, 2016. } \\
\text { Mohammadfam, Kamalinia, Momeni, } \\
\text { Golmohammadi, Hamidi, \& Soltanian, 2016; } \\
\text { Monzani, Espí-López, Zurriagac \& Andersen, } \\
\text { 2016; } \\
\text { Nowacki \& Bachnik, 2016; } \\
\text { Podgórski, 2015; } \\
\text { Rodríguez \& Molano, 2012; } \\
\text { Sheehan, Donohue, Shea, Cooper \& De Cieri, } \\
\text { 2016. }\end{array}$ \\
\hline
\end{tabular}




\begin{tabular}{|c|c|c|c|}
\hline Variables & Dimensiones & $\begin{array}{l}\text { Número de artículos } \\
\text { asociados a la dimensión }\end{array}$ & Estudios de soporte \\
\hline 1. Contexto interno & e) Calidad de vida en el trabajo & 9 & $\begin{array}{l}\text { Farid, Izadi, Arif \& Alipour, 2015; } \\
\text { González, Hidalgo \& Salazar, 2007; } \\
\text { González, Hidalgo, Salazar \& Peciado, 2010; } \\
\text { González, Hidalgo, Salazar \& Preciado, 2009; } \\
\text { Mohammad \& Narenji, 2011; } \\
\text { Parsa, Bin, Bin, Wahiza \& Parsa, 2014; } \\
\text { Safina, Kolesnikova, Karasik, Yurieva \& } \\
\text { Fakhrutdinova, 2015; } \\
\text { Shabbazi, Shokrzadeh, Bejani \& Malekinia, } \\
\text { 2011; } \\
\text { Shekh, Wan \& Abdul, 2014. }\end{array}$ \\
\hline & f) Regulación & 2 & $\begin{array}{l}\text { Arntz-Gray, 2016; } \\
\text { Kim, Park \& Park, } 2016 .\end{array}$ \\
\hline $\begin{array}{l}\text { 2. Contexto externo de } \\
\text { la organización }\end{array}$ & g) Competitividad & 6 & $\begin{array}{l}\text { Arntz-Gray, 2016; } \\
\text { Chang, Lin \& Wu, 2016; } \\
\text { Kim, Park \& Park, 2016. } \\
\text { Maier, Vadastreanu, Keppler, Eidenmuller \& } \\
\text { Maier, 2015; } \\
\text { Mohammadfam, Kamalinia, Momeni, } \\
\text { Golmohammadi, Hamidi \& Soltanian, 2016; } \\
\text { Wu, Chen \& Li, 2008. }\end{array}$ \\
\hline $\begin{array}{l}\text { 3. Enfoque de la } \\
\text { SST adoptado por la } \\
\text { organización }\end{array}$ & $\begin{array}{l}\text { h) Enfoque resolutivo } \\
\text { i) Enfoque preventivo } \\
\text { j) Enfoque promocional }\end{array}$ & 8 & $\begin{array}{l}\text { Arévalo \& Molano, 2013; } \\
\text { Budworth \& Khan, 2003 } \\
\text { Ergai, Cohen, Sharp, Wiegmann, } \\
\text { Gramopadhye \& Shappell, 2016; } \\
\text { Haslam, O'Hara, Kazi, Twumasi \& Haslam, } \\
\text { 2016; } \\
\text { Kim, Park \& Park, 2016; } \\
\text { Monzani, Espí-López, Zurriagac \& Andersen, } \\
\text { 2016; } \\
\text { Rodríguez \& Molano, 2012; } \\
\text { Waring, 2015. }\end{array}$ \\
\hline \multicolumn{2}{|c|}{ Número total de documentos } & $44^{*}$ & \\
\hline
\end{tabular}

Fuente: elaboración propia

* En total son 44 artículos, puesto que 9 de ellos aportan en varias dimensiones. 


\section{Contexto interno de la organización}

El análisis de contexto es la entrada para la identificación de las necesidades de la organización, así como de sus partes interesadas relevantes; provee información detallada basada en datos para la toma de decisiones en materia de la gestión de la organización (Rodríguez y Pedraza, 2016). Considerando los aspectos enunciados, se contemplan como elementos claves del contexto interno:

\section{a) Liderazgo de la alta dirección}

Se detectó que la alta dirección que cuenta con un fuerte compromiso con su gente y con la sociedad, en su conjunto puede hacer una diferencia sustancial en las organizaciones. Los factores que contribuyen al compromiso de la dirección hacia las prácticas de SST son la educación formal y el estímulo (Hossain, Hossain, Tarannum y Chowdhury, 2015).

El liderazgo tiene una influencia significativa en el bienestar y la satisfacción de los trabajadores. En este sentido, se demostró que se requiere de la aplicación de teorías como Full Range of Leadership Theory (FRLT) (Samad, Reaburn, Davis y Ahmed, 2015) y de estilos de liderazgo, de instrucción y persuasión, dado que estos fomentan la participación de los trabajadores y hacen que estos se sientan más integrados y comprometidos en las decisiones que tiene que ver con la forma en que hacen su trabajo diario (Chiang, Gómez y Salazar, 2014).

Los constructos teóricos que median la relación entre el sistema de GSST de una organización, su implementación y su éxito global son el liderazgo del grupo de trabajo, los valores organizacionales y las percepciones e interpretaciones de los trabajadores sobre el SGSST (Yorio, Willmer y Moore, 2015). La efectividad potencial de este sistema depende de: 1) la consistencia entre la estrategia y el desarrollo de las prácticas; 2) la representatividad y la relevancia de las prácticas en el riesgo real del trabajo; 3) la visibilidad y comprensibilidad del sistema de GSST al interior de la organización (Yorio, Willmer y Moore, 2015; Bowen y Ostroff, 2004).

\section{b) Participación y comunicación}

En la medida en que se logra una participación activa en la GSST se contribuye al empoderamiento de los líderes del tema en la organización (Grillo, 2013). La creación e implantación de un grupo de trabajo que participe en la toma de decisiones en SST es una muestra tangible de participación (Figueras, 2013). La participación está asociada a la comunicación en SST, la cual debe ser multidireccional, clara y efectiva. La comunicación es considerada como un riesgo que debe controlarse dentro de las organizaciones, pues se basa en diferentes medios y se debe dar en dos sentidos: top-down or bottom-up (Fekete, 2012).

Por otra parte, se encontró que la seguridad en el trabajo contribuye a la satisfacción general y a la salud psicofísica de los trabajadores, se encuentra relacionada con la motivación, aspecto esencial para el cambio en la conducta de los trabajadores (Hedlund, Gummesson, Rydell y Andersson, 2016). Se evidenció que ante una situación de emergencia los individuos generan un comportamiento colectivo social, el cual es ordenado y cooperativo (Fang, El-Tawil y Aguirre, 2016). Según Živkovic (2013) los principales motivadores de los trabajadores son: 1) salario; 2) motivos de seguridad laboral; 3) seguridad y salud en el trabajo. Para aumentar la motivación, los objetivos deben ser comunes, ambiciosos y claros. El líder debe potenciar actitudes y crear una sensación de logro y de pertenencia a la organización (Hedlund, Gummesson, Rydell y Andersson, 2016). 


\section{c) Aprendizaje y desarrollo de la cultura de los individuos}

Las empresas deben innovar en el diseño, implementación y mantenimiento de sus estructuras y sistemas de gestión del conocimiento para contribuir al logro de sus resultados (Nowacki y Bachnik, 2016). Esto requiere hacer uso del aprendizaje organizacional; según Weick y Sutcliffe (2015) los tomadores de decisiones necesitan desarrollar capacidades que les permitan conocer detalles e información relevante para crear orden y fomentar la previsibilidad.

Las organizaciones deben medir la propensión a aprender en materia de seguridad. Para ello se diseñaron: 1) indicadores organizacionales, orientados a la identificación de las fortalezas, debilidades y formas de mejora como parte de los indicadores de seguridad; 2) indicadores individuales, que propenden por la identificación de las personas que puedan desempeñar el papel de "agentes de aprendizaje" (Drupsteen y Wybo, 2015).

Otro aspecto a considerar es la propensión por el esfuerzo. Según Schnake (2007), son siete los efectos positivos directos que conlleva el esfuerzo: satisfacción en el trabajo, capacidad de trabajo, compromiso con la organización, rasgos de personalidad, capacidad para realizar tareas relacionadas con el trabajo, normas de comportamiento del grupo y el tamaño del grupo y la moderación por el temor a la evaluación. Es de precisar que el fin del aprendizaje en la organización es la creación de una cultura, puesto que una cultura de seguridad débil conlleva a accidentes graves (Warszawska y Kraslawski, 2015). De acuerdo con Martins y Coetzee (2007), la cultura organizacional es un patrón integrado de comportamiento, el cual dirige la organización al logro de metas; y según Martins y Nel (2014), la SST puede tener un impacto en la cultura organizacional y viceversa.

\section{d) Innovación, medición y mejora continua}

La GSST requiere de procesos de innovación (Maier, Vadastreanu, Keppler, Eidenmuller y Maier, 2015) que den cuenta de las distintas formas con las cuales las organizaciones puedan abordar los nuevos procesos de salud-enfermedad en la población trabajadora. La gestión del conocimiento es un canal para la innovación que permite el desarrollo de estrategias para capturar y asegurar las ventajas competitivas de las organizaciones (Nowacki y Bachnik, 2016). En este sentido, según Bernardo (2014), existen dos tipos de innovación: radical e incremental.

En la propuesta de modelo de GSST desarrollada por Rodríguez (2012), se identifican dentro de sus dimensiones la evaluación y la mejora continua. La evaluación considera la medición y análisis del desempeño, las acciones correctivas y preventivas. Para ello, los indicadores deben dar cuenta de la evolución de las actividades y de su impacto sobre la población trabajadora y no centrarse exclusivamente en la presencia de eventos negativos como incidentes, accidentes, enfermedades profesionales, incapacidades, sino que deben considerarse aquellos dirigidos a la prevención y a otros aspectos que en la actualidad no son tangibles, como el presentismo laboral (Rodríguez, 2012; Monzani, Espí-López, Zurriagac y Andersen, 2016; Sheehan, Donohue, Shea, Cooper y De Cieri, 2016).

Podgórski (2015), plantea indicadores para la evaluación de la conformidad del sistema, a través de indicadores de desempeño estructural del sistema y de indicadores de desempeño operacional (evaluación de la efectividad de los procesos internos del sistema). Los indicadores que se espera contemple una organización dentro de su GSST son: indicadores de selección y agregación, indicadores globales, e indicadores de análisis MCDM (multi-criteria decision making). Según 
Carlucci (2010), la selección de los indicadores debe considerar la pertinencia, fiabilidad, comparabilidad y coherencia, comprensibilidad y la calidad de la representación. Para Mohammadfam et al. (2016), las organizaciones deben plantear indicadores clave de GSST, con el ánimo de garantizar una gestión estructurada y sistemática (política, planeación, implementación y operación, revisión y revisión por la dirección) para el control del riesgo en SST.

En el estudio de Mansour (2016), se plantea la cuantificación de costos intangibles relacionados con el trabajo no ergonómico y las lesiones derivadas del trabajo que están asociadas a los niveles de estrés entre los empleados. Estas condiciones representan "costos ocultos" que las organizaciones no contemplan a pesar del impacto que tiene en su rendimiento. En otras palabras, existen costos tangibles y de los intangibles derivados de las condiciones de trabajo y de las lesiones/enfermedades que estas generan en los trabajadores.

En cuanto a la mejora continua, esta requiere de la información derivada de la dimensión de evaluación para la toma de decisiones en el sistema de GSST. Esta dimensión implica la participación y revisión por parte de la dirección. El ajuste que se derive de esta revisión se verá reflejado en las acciones correctivas, preventivas y de mejora que se ejecuten (Rodríguez, 2012).

\section{e) Calidad de vida en el trabajo}

El concepto de calidad de vida en el trabajo (CVT), no es reciente, sin embargo, mejorar el nivel de CVT en una de las prioridades para las organizaciones (Safina, Kolesnikova, Karasik, Yurieva y Fakhrutdinova, 2015). De acuerdo con Mohammad y Narenji (2011) la CTV tiene una estructura múltiple dinámica que envuelve conceptos como seguridad en el trabajo, sistemas de recompensa, flujos de trabajo, oportunidades de educación y desarrollo laboral; además, la participación en la toma de decisiones. Es un término que contiene características de subjetividad y multidimensionalidad, aspectos que dependen de las creencias y de los valores de los individuos, de su contexto cultural y personal (González, Hidalgo y Salazar, 2007). Para Shekh, Wan y Abdul (2014), la salud, el bienestar y la CVT se refieren a aspectos físicos y psicológicos de un individuo en un entorno de trabajo.

La CVT contempla aspectos de soporte institucional, seguridad e integración al puesto de trabajo y satisfacción por el mismo, bienestar conseguido a través de su actividad laboral, desarrollo personal logrado y la administración de su tiempo libre (González, Hidalgo, Salazar y Peciado, 2010). Shahbazi, Shokrzadeh, Bejani y Malekinia (2011), consideran como dimensiones de la CVT la compensación adecuada y justa, las condiciones de trabajo seguras y saludables, la oportunidad inmediata para usar y desarrollar las capacidades humanas, la oportunidad para el crecimiento continuo y la seguridad, la integración social en la organización del trabajo, el constitucionalismo en la organización del trabajo, el trabajo mismo y la vida total de espacio y relevancia social de la vida laboral.

Según Farid, Izadi, Arif y Alipour (2015), la CTV contempla ocho dimensiones, a saber: indemnización justa y adecuada, condiciones de trabajo, el uso y desarrollo de las capacidades, posibilidades de crecimiento y la seguridad, la integración social, el constitucionalismo, el trabajo y el espacio total de la vida y la relevancia social. Por otra parte, el compromiso organizacional fue analizado desde tres orientaciones: compromiso afectivo, compromiso normativo y el compromiso de continuidad. La CVT también es asumida como el entorno de la organización, de acuerdo con las necesidades (salud y seguridad, económicas y familiares, sociales, autoestima, actualización, conocimiento y estéticas) de 
los empleados. Se encontró relación estadísticamente significativa entre la CVT y la promoción profesional de los empleados académicos, aspecto que aumenta el valor de las organizaciones (Parsa, Bin, Bin, Wahiza y Parsa, 2014).

\section{Contexto externo de la organización}

En este apartado se desarrollan los factores externos, los cuales de acuerdo con el modelo propuesto por Hossain, Hossain, Tarannum y Chowdhury (2015) corresponden a la legislación, competitividad y a la mimética; sin embargo, en esta investigación se asumen los dos primeros debido a que se logró recopilar evidencia científica que argumenta su objetividad.

\section{f) Regulación}

Se ha evidenciado que el cumplimiento de la normativa en SST hace parte del sistema de responsabilidad interna de las organizaciones; además, estas deben atender a los requerimientos de la legislación externa de acuerdo con las condiciones establecidas en cada país. Sin embargo, más allá del cumplimiento de las leyes y de los reglamentos, se deben considerar otras herramientas que fomenten la equidad y la sostenibilidad de las políticas globales de la organización, las cuales deben estar basadas en el cambio y en enfoques de mando y control (Arntz-Gray, 2016).

En términos generales, se encontró que los sistemas de GSST y la cultura de prevención han tenido una evolución de acuerdo con las transformaciones normativas tanto en el ámbito nacional como en los ámbitos organizacionales. De acuerdo con el estudio de Kim, Park y Park (2016), se evidencia que en la medida en que se logre una cultura de seguridad positiva, la tasa de incidentes tiende a reducirse, aspecto relacionado históricamente con el desarrollo de políticas nacionales que integran tres elementos, a saber: tecnología, sistemas de GSST e influencia en la cultura de seguridad.

\section{g) Competitividad}

Las organizaciones requieren ser competitivas y una de las estrategias que han emprendido para demostrar este atributo son los procesos de certificación en sistemas de gestión, dado que a través de estos aumentan la probabilidad de obtención negocios y por ende el desarrollo de la organización; desarrollan habilidades en los trabajadores y estrategias para la adaptación de los cambios en tecnologías, mejoran su imagen, estatus social y el reconocimiento público (Chang, Lin y $\mathrm{Wu}$, 2016). Además, la práctica de SST da un estatus más alto, otorga mayor prestigio, simboliza el estado de la industria, es una señal de ser competente (Wu, Chen y Li, 2008; Hossain, Hossain, Tarannum y Chowdhury, 2015).

De acuerdo con el estudio de Kim, Park y Park (2016), se demostró que la inclusión de estrategias para el fomento de la cultura de seguridad, así como las etapas para su mejora, inciden positivamente en la reducción de la accidentalidad de los trabajadores, estos son aspectos que contribuyen a la sostenibilidad del negocio. En este sentido, de acuerdo con Maier, Vadastreanu, Keppler, Eidenmuller y Maier (2015) es necesario crear nuevos métodos y herramientas para poder innovar en la gestión, dado que esto es indispensable para mantener las ventajas competitivas e incrementar la satisfacción de clientes y trabajadores.

Para evidenciar el impacto del sistema de GSST sobre la SST de los trabajadores, se requiere de un enfoque de toma de decisiones que integra dos técnicas: Analytical Network Process (ANP) y Technique for Order Preference by Similarity to Ideal Solution (TOPSIS), para evaluar y mejorar la efectividad del estándar OHSAS 18001. Se encontró que los factores más influyentes que deben tenerse en cuenta para mejorar la efectividad de la 
norma OHSAS 18001 son el compromiso de la dirección, la participación de los trabajadores, la asignación de los recursos financieros, la formación, la evaluación de riesgos, la responsabilidad definitiva, la comunicación y la difusión de las actividades y de los resultados de salud y seguridad ocupacional (Mohammadfam $e t$ al., 2016).

\section{Enfoque de la SST adoptado por la organización}

La SST surgió como parte de la evolución de la salud ocupacional. Según Arévalo y Molano (2013), la SST y su enfoque se ha modificado en la medida en que está articulado a distintas disciplinas, entre ellas la sociología y la ergonomía. En este sentido, como se puede apreciar en la figura 1, la gestión de los riesgos laborales tuvo un carácter resolutivo, y en la medida en que este concepto ha evolucionado, las intervenciones se han orientado hacia enfoques preventivos y promocionales. En la actualidad, las organizaciones deben replantear su gestión pasando de un enfoque resolutivo a uno promocional (Monzani, Espí-López, Zurriagac y Andersen, 2016).

En el estudio de Haslam, O'Hara, Kazi, Twumasi y Haslam (2016), se reconocen tres enfoques de la SST: 1) cuando las organizaciones no están completamente relacionadas con la SST; 2) organizaciones que cumplen con los requisitos de SST; 3 ) organizaciones proactivas en la gestión de la SST (Haslam, O'Hara, Kazi, Twumasi y Haslam, 2016).

Aunado a ello, se le ha dado fuerza al concepto de cultura de prevención, el cual, de acuerdo con lo expresado por Kim, Park y Park (2016), está implícitamente basado en el concepto de cultura de seguridad, dado que ambos utilizan un enfoque de la siguiente manera: 1) la cultura de prevención tiene como objetivo reducir los riesgos relacionados o no con el trabajo, se ubica en un ámbito social y nacional, por cuanto se orienta a la protección y a la promoción de la SST, además, requiere de la participación del gobierno en su conjunto para garantizar que la SST de los trabajadores sea una prioridad en la agenda nacional; 2) la cultura de seguridad se encarga exclusivamente de los riesgos asociados al lugar de trabajo y hace énfasis en la protección de la SST. Se ubica en un ámbito organizacional principalmente, además de la participación del Ministerio de Trabajo.

El abordaje de la SST debe darse de manera integral, considerando factores ambientales, condiciones de la operación, factores del personal, factores relacionados con la supervisión y factores de influencia organizacional como son los procesos, el clima organizacional y los recursos para la gestión (Ergai et al., 2016).

En este sentido, Waring (2015) plantea la necesidad de adoptar un nuevo sistema integrado para examinar la seguridad, este debe combinar los puntos fuertes de la gama de disciplinas pertinentes de una manera holística, logrando con ello superar sus debilidades individuales. Haslam, O'Hara, Kazi, Twumasi y Haslam (2016), consideraron el modelo de Continuous Improvement Cycle desarrollado por Budworth y Khan (2003) junto con las actitudes, las condiciones de organización y los sistemas para clasificar a las organizaciones de acuerdo con los siguientes aspectos: 1) las actitudes hacia la SST; 2) las prioridades de gestión de la SST; 3) compromiso de la dirección; 4) participación de los interesados; 5) la formación y la comunicación; 6) la mejora de la SST; 7) formalización de gestión de la SST. 
Figura 1. Enfoque de la SST de acuerdo con su evolución conceptual

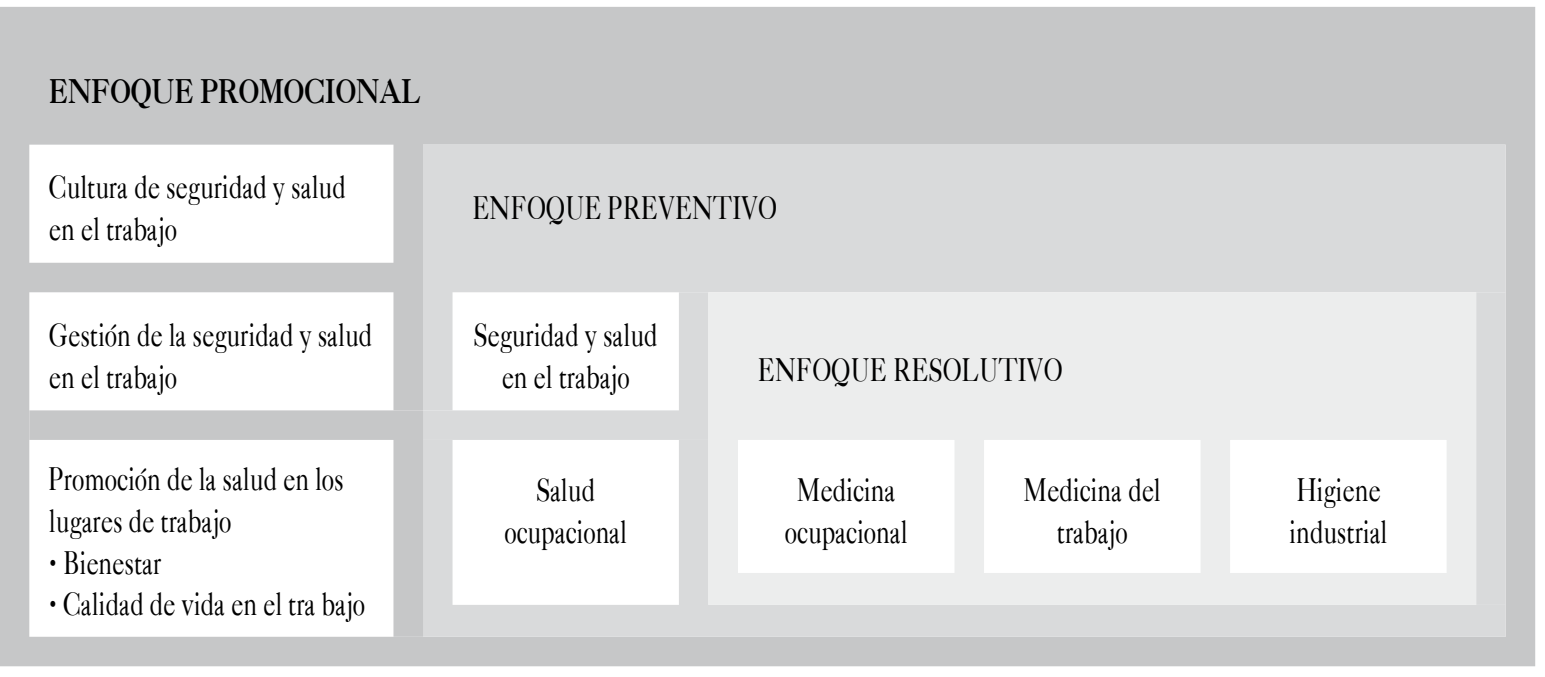

Fuente: elaboración propia (2017)

\section{CONCLUSIONES}

La revisión de literatura permitió identificar tres variables (contexto interno, contexto externo y el enfoque de la seguridad y salud en el trabajo -concebido como una estrategia central de la organización-) y diez dimensiones base para la evaluación de la madurez de la GSST en las organizaciones. Hallazgos que coinciden con lo planteado por Kluth, Jäger, Schatz y Bauernhansl (2014), quienes señalan que la madurez incluye los niveles de habilidad de la organización, por cuanto proporciona elementos y medidas definidas. $\mathrm{La}$ madurez implica un progreso evolutivo en la demostración de habilidades específicas para lograr objetivos, desde un estado inicial en el que se consideran unos conocimientos relacionados con la complejidad de la gestión hasta un estado final, que completa la optimización de los recursos de empresa para alcanzar los objetivos de armonización de la complejidad interna y externa de la organización. En este sentido, los aspectos contemplados en la conceptualización de estos autores fueron incluidos y soportados teóricamente en las variables y dimensiones descritas.
Adicionalmente, la revisión evidenció un amplio interés académico e investigativo en el concepto de madurez de la GSST en la última década, aspecto que se soporta en que la mayoría de las publicaciones se ubican en este periodo. Aunque se lograron definir diez dimensiones para evaluar la madurez de esta gestión, se requiere dar continuidad a la investigación con el desarrollo de instrumentos y escalas confiables que permitan medir la madurez de esta gestión en las organizaciones.

De acuerdo con lo planteado por Domingues, Sampaio y Arezes (2014a) se requiere establecer relaciones cualitativas y cuantitativas entre variables para explicar cómo estas afectan a la variable latente, es decir, el nivel de madurez del objeto maduración. Lo anterior con el fin efectuar un análisis de contexto integrado, puesto que se consideran las acciones de mejora y los aportes de otros subsistemas de gestión implementados. En este sentido, un modelo que evidencie la madurez de los sistemas integrados de gestión debe contemplar tres variables: la visión amplia de la alta dirección, la clasificación de los niveles de integración y 
los tipos de auditoría (Domingues, Sampaio y Arezes, 2014b; Moumen y Aoufir, 2016).

En otras palabras, las variables y dimensiones de la madurez identificadas en la revisión de la literatura, se constituyen en un insumo para la definición de una secuencia de niveles que reúnen un conjunto de características de la gestión. Estos niveles constituyen y anticipan caminos lógicos que llevan a la organización de un estado inicial a un nivel de madurez en la organización, por cuanto la GSST debe integrarse en la gestión global de la organización.

En términos generales, el medir y categorizar la madurez de la GSST facilita la búsqueda de oportunidades, riesgos y vulnerabilidades, la identificación de estrategias para la mejora, un mejor control de los costos y tiempos y la introducción de actividades de gestión de acuerdo con la complejidad de las organizaciones, considerando la versión anterior, actual y futura de la GSST, como referentes para la identificación de una versión más predecible y precisa que dé respuesta a las necesidades y las estrategias de negocio de la organización en cuestión.

\section{REFERENCIAS}

Arévalo, N., y Molano, J. (2013). De la salud ocupacional a la gestión de la seguridad y salud en el trabajo: más que semántica, una transformación del sistema general de riesgos laborales. Innovar, 23(48), 21-32.

Arntz-Gray, J. (2016). Plan, do, check, act: The need for independent audit of the internal responsibility system in occupational health and safety. Safety Science, 84, 12-23.
Bernardo, M. (2014). Integration of management systems as an innovation: A proposal for a new model. Journal of Cleaner Production, 82, 132-142.

Bowen, D., \& Ostroff, C. (2004). Understanding HRM-Firm performance linkages: The role of the "Strength" of the HRM system. Academic of Management Review, 29(2), 203-222.

Budworth, N., \& Khan, S. (2003). Climbing the occupational health and safety mountain and taking your team with you. Safety and Health Practitioner, 21, 30-33.

Carlucci, D. (2010). Evaluating and selecting key performance indicators: an ANP- based model. Measuring Business Excellence, 14(2), 66-76.

Chang, S., Lin, C., \& Wu, W. (2016). The features and marketability of certificates for occupational safety and health management in Taiwan. Safety Science, 85, 77-87.

Chen, S., Yu, D., \& Zheng, X. (2014). Maturity evaluation on safety standardization in hydropower construction: Methodology and case study. Engineering Letters, 22(4), 194-201.

Chiang, M., Gómez, N., y Salazar, C. (2014). Satisfacción laboral y estilos de liderazgo en instituciones públicas y privadas de educación en Chile. Cuadernos de Administración, 30(52), 65-74.

Cliff, D. (2012). The management of occupational health and safety in the Australian mining industry. Australia: International mining for development centre.

Domingues, J., Sampaio, P., \& Arezes, P. (2014a). A model for assessing maturity of Integrated Management Systems. In P. Arezes, J. Baptista, M. Barroso, P. Carneiro, P. Cordeiro, N. Costa, G. Perestrelo 
(Ed.). Occupational Safety and Hygiene II, 818. London: CRC Press.

Domingues, J., Sampaio, P., \& Arezes, P. (2014b). Maturity models: A useful solution to assess current OHS management system. Braga: Institution of Occupational Safety and Health.

Drupsteen, L., \& Wybo, J. (2015). Assessing propensity to learn from safety-related events. Safety Science, 71, 28-38.

Ergai, E., Cohen, T., Sharp, J., Wiegmann, D., Gramopadhye, A., \& Shappell, S. (2016). Assessment of the human factors analysis and classification system (HFACS): Intra-rater and inter-rater reliability. Safety Science, 82, 393-398.

Fang, J., El-Tawil, S., \& Aguirre, B. (2016). Leader-follower model for agent based simulation of social collective behavior during egress. Safety Science, $83,40-47$.

Farid, H., Izadi, Z., Arif, I., \& Alipour, F. (2015). Relationship between quality of work life and organizational commitment among lecturers in a Malaysian public research university. The Social Science Journal, 52(1), 54-61.

Fekete, A. (2012). Safety and security target levels: Opportunities and challenges for risk management and risk communication. International Journal of Disaster Risk Reduction, 2, 67-76.

Ferreira, T., \& Simões, C. (2015). Assessment of maturity in project management: A bibliometric study of main models. Procedia Computer Science, 55, 92-101.

Figueras, F. (2013). Liderazgo de la dirección y participación de los trabajadores en el ámbito de la seguridad y salud en el trabajo. Medicina y Seguridad del Trabajo, 59, 1-59.

Foster, P., \& Hoult, S. (2013). The safety journey: Using a safety maturity model for safety planning and assurance in the UK coal mining industry. $\mathrm{Mi}$ nerals, 3, 59-72.

González, R., Hidalgo, G., y Salazar, J. (2007). Calidad de vida en el trabajo: un término de moda con problemas de conceptuación. Psicología y Salud, 17, 115-123.

González, R., Hidalgo, G., Salazar, J., y Preciado, M. (2010). Elaboración y validación del instrumento para medir calidad de vida en el trabajo "CVTGOHISALO”. Ciencia y Trabajo, 12(36), 332-340.

González, R., Hidalgo, G., Salazar, J., y Preciado, M. (2009). Instrumento para medir la calidad de vida en el trabajo (CVT-GOHISALO): manual para su aplicación y su interpretación. Guadalajara, México: Editorial de la Noche.

Grillo, N. (2013). Construcción y validación de una herramienta de gestión para evaluar la cultura de seguridad en entornos industriales. Barcelona: Universitat Ramon Llull.

Haslam, C., O'hara, J., Kazi, A., Twumasi, R., \& Haslam, R. (2016). Proactive occupational safety and health management: Promoting good health and good business. Safety Science, 81, 99-108.

Hedlund, A., Gummesson, K., Rydell, A., \& Andersson, M. (2016). Safety motivation at work: Evaluation of changes from six interventions. Safety Science, 82, 155-163.

Hossain, M., Hossain, M., Tarannum, S., \& Chowdhury, T. (2015). Factors affecting OHS practices 
in private universities: An empirical study from Bangladesh. Safety Science, 72, 371-378.

Kim, Y., Park, J., \& Park, M. (2016). Creating a culture of prevention in occupational safety and health practice. Safety and Health at Work, 7(2), 89-96.

Kluth, A., Jäger, J., Schatz, A., \& Bauernhansl, T. (2014). Evaluation of complexity management systems - systematical and maturity-based approach. Procedia CIRP, 17, 224-229.

Maier, D., Vadastreanu, A., Keppler, T., Eidenmuller, T., \& Maier, A. (2015). Innovation as a part of an existing integrated management system. Procedia Economics and Finance, 26, 1060-1067.

Mansour, M. (2016). Quantifying the intangible costs related to non-ergonomic work conditions and work injuries based on the stress level among employees. Safety Science, 82, 283-288.

Martins, N., \& Coetzee, M. (2007). Organizational culture, employee satisfaction, perceived leader competency and personality Type: An exploratory study in a South African engineering company. Journal of Human Resources, 5(2), 20-32.

Martins, N., \& Nel, M. (2014). Validating a theoretical model of organizational culture and occupational health by means of structural equation modelling. In proceedings of the 13 European conference on research methodology for business and management studies. London: Academic Conferences Limited.

Mohammadfam, I., Kamalinia, M., Momeni, M., Golmohammadi, R., Hamidi, Y., \& Soltanian, A. (2016). Developing an integrated decision making approach to assess and promote the effectiveness of occupational health and safety management systems. Journal of Cleaner Production, 127, 119-133.

Monzani, L., Espí-López, G., Zurriagac, R., \& Andersen, L. (2016). Manual therapy for tension-type headache related to quality of work life and work presenteeism: Secondary analysis of a randomized controlled trial. Complementary Therapies in Medicine, 25, 86-91.

Moumen, M., \& Aoufir, H. (2016). Evaluation of maturity level of QSE management systems: Empirical analysis, case of Moroccan companies. Modern Applied Science, 10(5), 1913-1852.

Nowacki, R., \& Bachnik, K. (2016). Innovations within knowledge management. Journal of Business Research, 69(5), 1577-1581.

Parsa, B., Bin, K., Bin, B., Wahiza, N., \& Parsa, P. (2014). Relationship between quality of work life and career advancement among Iranian academics. Procedia-Social and Behavioral Sciences, 152, 108-111.

Podgórski, D. (2015). Measuring operational performance of OSH management system. A demonstration of AHP-based selection of leading key performance indicators. Safety Science, 73, 146-166.

Rodríguez, Y. (2012). Estrategias para el mejoramiento de la gestión de la salud y seguridad en el trabajo frente a las formas de vinculación en plantas de un grupo empresarial del sector industrial de Bogotá D. C. (Trabajo de maestría). Bogotá: Universidad Nacional de Colombia.

Rodríguez, Y., y Molano, J. (2012). Adaptación de una herramienta para la evaluación de la gestión de la salud y seguridad en el trabajo. El Hombre y la Máquina, 40, 7-21. 
Rodríguez, Y., \& Pedraza, X. (2016). Aportes de la estructura de alto nivel a la gestión integrada. Revista Global de Negocios, 5(2), 65-75.

Safina, L., Kolesnikova, J., Karasik, E., Yurieva, O., \& Fakhrutdinova, A. (2015). The higher education impact on the quality of young people working life. Procedia-Social and Behavioral Sciences, 191, 2412-2415.

Samad, A., Reaburn, P., Davis, H., \& Ahmed, E. (2015). Towards an understanding of the effect of leadership on employee wellbeing and organizational outcomes in australian universities. The Journal of Developing Areas, 49(6), 442-448.

Schnake, S. (2007). An integrative model of effort propensity. Human Resource Management Review, 17, 274-289.

Shahbazi, B., Shokrzadeh, S., Bejani, H., \& Malekinia, E. G. (2011). A Survey of relationship between the quality of work life and performance of Department Chairpersons of Esfahan University and Esfahan Medical Science University. ProcediaSocial and Behavioral Sciences, 30, 1555-1560.

Sheehan, C., Donohue, R., Shea, T., Cooper, B., \& De Cieri, H. (2016). Leading and lagging indicators of occupational health and safety: The moderating role of safety leadership. Accident Analysis and Prevention, 92, 130-138.

Shekh, A., Wan, W., \& Abdul, A. (2014). Motivations using social networking sites on quality work life. Procedia-Social and Behavioral Sciences, 130, $524-531$.

Tahri, T., \& Drissi-Kaitouni, O. (2015). New design for calculating Project Management Maturity (PMM). Procedia-Social and Behavioral Sciences, 181, 171-177.

Waring, A. (2015). Managerial and non-technical factors in the development of human-created disasters: A review and research agenda. Safety Science, 79, 254-267.

Warszawska, K., \& Kraslawski, A. (2015). Method for quantitative assessment of safety culture. Journal of Loss Prevention in the Process Industries, 42, 27-34.

Weick, K., \& Sutcliffe, K. (2015). Managing the Unexpected: Sustained Performance in a Complex World. New Jersey, USA: John Wiley \& Sons, Inc.

Wu, T., Chen, C., \& Li, C. (2008). A correlation among safety leadership, safety climate and safety performance. Journal of Loss Prevention in the Process Industries, 21(3), 307-318.

Yorio, P., Willmer, D., \& Moore, S. (2015). Health and safety management systems through a multilevel and strategic management perspective: Theoretical and empirical considerations. Safety Science, 72, 221-228.

Živkovic, S. (2013). Management of occupational safety by motivating employees to work safely. African Journal of Business Management, 7(12), 936-946. 